\title{
A UNIFIED PICTURE OF DISK GALAXIES WHERE BARS, SPIRALS AND WARPS RESULT FROM THE SAME FUNDAMENTAL CAUSES
}

\author{
Daniel Pfenniger and Yves Revaz \\ Geneva Observatory, University of Geneva, CH-1290 Sauverny, Switzerland
}

\begin{abstract}
Bars and spiral arms have played an important role as constraints on the dynamics and on the distribution of dark matter in the optical parts of disk galaxies. Dynamics linked to the dissipative nature of gas, and its transformation into stars provide clues that spiral galaxies are driven by dissipation close to a state of marginal stability with respect to the dynamics in the galaxy plane. Here we present numerical evidences that warps play a similar role but in the transverse direction. N-body simulations show that typical galactic disks are also marginally stable with respect to a bending instability leading to typical observed warps. The frequent occurrence of warps and asymmetries in the outer galactic disks give therefore, like bars in the inner disks, new constraints on the dark matter, but this time in the outer disks. If disks are marginally stable with respect to bending instabilities, our models suggest that the mass within the HI disks must be a multiple of the detected HI and stars, i.e., disks must be heavier than seen. But the models do not rule out a traditional thick halo with a mass within the HI disk radius similar to the total disk mass.
\end{abstract}

Keywords: Bars, spiral arms, warps, dark matter, galaxy dynamics

\section{Introduction}

Over the years we discover progressively the true nature of galaxies by confronting observations to theory and vice versa. A simple tool to rank the importance of the various physical ingredients at play in galaxies is a more complete form of the virial theorem than usually discussed:

$$
\frac{1}{2} \ddot{I}=\underbrace{2 E_{\text {kin }}}_{>0}+\underbrace{E_{\text {grav }}}_{<0}+\underbrace{3 P_{\text {int }} V}_{>0} \underbrace{-3 P_{\text {ext }} V}_{<0}+\underbrace{E_{\text {mag }}}_{>0} \cdots \approx 0 .
$$

It allows to rank the main energies (bulk kinetic, gravitational, internal and external pressures, magnetic, etc.) determining the system equilibrium measured by its moment of inertia $I$ in the volume $V$. Clearly the magnitudes of the interacting energies (mainly bulk kinetic against gravitational energy in galax-

D R A F T Page 1 February 7, 2020, 5:59pm D R A F T 
ies) rank the importance of each factor. A Taylor expansion of this equation in time also shows that an evolution along a sequence of quasi-steady equilibria is determined to first order by the magnitude of the interacting powers (mainly gas cooling against mechanical heating power).

The main point emphasized here is that the same rules found to well explain to first order the horizontal properties of spiral galaxies, i.e., gravitational physics supplemented by energy dissipation, are also able to explain the ubiquitous warps. By comparing numerical simulations of thin self-gravitating disks to the observed properties of spirals, in particular to their frequent warps, a coherent dynamical and evolutive picture of spiral galaxies emerges, with the suggestive hint that two types of dark matter are involved: 1) the classical extended dark halos much thicker than the disks, and 2) a dark component coeval with the HI disks similar to the one proposed in Pfenniger \& Combes (1994).

Nowadays the need of at least two types of dark matter is actually well motivated. From the big-bang predicted baryogenesis most of the baryons remain to be found, and on the other hand, also from cosmology non-baryonic dark matter is required to obtain a coherent description of large scale structure formation and the Universe cosmological parameters. Since baryons are known to be strongly dissipative and sometimes collisional, contrary to the expected non-baryonic dark matter, we have no grounds to expect that in galaxies their respective spatial distributions should coincide.

\section{The role of bars and spirals}

The bar instability has been used in the 70's for supporting the idea that an extended dark matter halo must exist to prevent bar formation (Ostriker \& Peebles, 1973). Indeed the early N-body simulations showed that bars result spontaneously from a dynamical plane instability in a collisionless disk with an initial flat core. But because theoreticians were wrongly assuming that bars were exceptional, they imagined a hot and massive collisionless component coexisting in the optical disks as a solution to prevent the quick formation of bars, despite the awareness by skilled observers such as de Vaucouleurs that bars are frequent. Subsequent higher resolution and infrared observations revealed that bars are in fact even more frequent and found in a majority of spirals, as reminded in several papers at this conference.

The reverse problem was thus discussed many times: how bars and hot dark halos can coexist, since dark halos were then no longer viewed as hypothetical. It was also understood over the years that barless disks can exist when the central density profile is too centrally concentrated.

Spiral density waves are a more general but less robust version of the bar phenomenon. The main reason for spiral formation is now well understood as resulting from the non-linear growth of a spontaneous gravitational instability

D R A F T Page 2 February 7, 2020, 5:59pm D R A F T 
in the disk plane with the same origin as for bars: a kinematically too cold disk is gravitationally unstable, and the non-linear result is typically a bar in the initial flat core and spiral arms in the outer differentially rotating region. Without invoking more than Newtonian physics it was also found that the typical double exponential disks profiles are also a natural asymptotic state for a collisionless disk passing through a bar instability (Pfenniger \& Friedli, 1991).

The non-linear structures resulting from the gravitational instability are never strictly stationary, but evolve secularly (over several rotational periods). In pure collisionless disks they tend to destroy the spiral arms and later bars, so obviously something must regenerate them in real galaxies.

The theory of bars and spirals is presently incomplete because the full selfconsistent problem is very non-linear. Thus no analytical theory able yet to predict the full development of strongly rotating collisionless self-gravitating disks. Only the brute force N-body simulations are able to do it.

Since the Newtonian physics involved in these $\mathrm{N}$-body experiments is very well understood, and that the numerical codes can to a large extent be trusted because different versions implementing different techniques have been developed and checked over several decades by many groups, we can use these Nbody techniques to predict and explain the behaviours of galaxies. The results of such N-body simulations should be taken as seriously as analytical developments in celestial mechanics. As example of success of N-body techniques is the prediction that bars may evolve into peanut-shaped structures. This was first empirically found in N-body experiments (Combes \& Sanders, 1981; Combes et al., 1990), understood theoretically (Pfenniger \& Friedli, 1991), and later confirmed by observations (e.g., Bureau \& Freeman, 1999).

Coupled to this well understood underlying physics, numerous independent studies of the mass to light ratio in the optical parts of spirals have determined that a substantial fraction of the gravitating mass there is well explained by the detected baryons (e.g. Sancisi, 2003). This ensures that we have at least a basic physical understanding of the inner parts of galaxies.

\section{The role of gas}

Since disk galaxies as star producing systems must contain also a lot of gas, its effects must be considered on the long run. First, dust polluted gas is very efficient to lose its thermal energy by infrared radiation, so galaxies must be seen, besides in first approximation as rotating self-gravitating objects, in second approximation also as energy dissipating structures. Gravitationally bound rotating structures slowly losing energy tend to rapidly converge towards thin disks because then angular momentum is a quantity much harder to dissipate away than energy.

D R A F T Page 3 February 7, 2020, 5:59pm D R A F T 
So the frequent occurrence of spiral arms (after all disk galaxies are called spirals!) follows directly from the constant competition between the effect of cooling driving disks towards the gravitational Safronov-Toomre instability threshold. Any further cooling leads to strong reaction from the disk by dynamical heating. As long as gas cooling continues to be efficient the natural long term state of spiral galaxies is therefore to stay close to the marginal stability threshold.

Numerous studies show that galactic disks, including the Milky Way, have disks close to a marginal stability state with a Safronov-Toomre parameter $Q$ close to unity. Because of this marginal state, spiral galaxies do react strongly to other perturbations such as galaxy interactions by amplifying spiral arms.

Many studies have been trying to determine whether spirals and bars result either from galaxy interactions or from a proper disk instability. The more fundamental cause of spirals and bars is actually the internal marginal stability state making galactic disks very reactive to various perturbations. Even small satellite interactions trigger large responses from a disk in the form of grand design spirals. The name of "spirals" for disk galaxies is in the end an excellent way to characterize their close to marginal stability state, showing both that dissipation acts and dynamics reacts.

A corollary of such a marginally stable state is that a steady state is unlikely. Instead evolution is to be expected as long as the marginal stability state is maintained by the competing factors, gas cooling against dynamical heating.

As by-product the large scale dynamical instability of galactic disks leads to local interstellar gas compression, shocks and turbulence, cascading down to smaller scale gas instabilities (e.g., Fleck, 1981; Elmegreen, 2004). At the bottom of the cascade the most visible effect of the gas "turbulence" is star formation (Klessen, 2004), which implies gas consumption. The long term effects of large scale instabilities is to transform progressively the dissipative component into a collisionless stellar component. By consuming gas the cooling agent becomes rarer, and by forming stars the dynamical heating more effective in counter-balancing gas cooling. In addition the mechanical energy output produced especially by massive stars provides a second important source of heating competing gas cooling.

\section{Constraints on dark matter forms}

So the slow transformation of matter from gas rich, but also dark matter rich disks to gas poor, star rich and dark matter poor structures already indicates that the above picture is broadly consistent. Gas poor disk galaxies (S0's, Sa's) have namely typically less prominent and open spiral arms in more symmetric disk, while gas rich spirals (Sd's, Sc's) have large open spirals in irregular disks. The fact that along the spiral sequence the visible gas represents always

D R A F T Page 4 February 7, 2020, 5:59pm D R A F T 
a minor fraction of the mass indicates that some of the dark mass must be gas in order to be able to form subsequently all the stars that are seen in S0's and Sa's (Pfenniger et al., 1994).

However the fact that S0's and Sa's still contain a fraction of dark mass while showing very little star formation indicates also that some of the dark mass is in a form that cannot easily form stars. Around $40 \%$ of the total mass within the HI disk radius might be in a dark collisionless form. Therefore the above considerations show already that we can have a consistent dynamical picture of disk galaxies including the gas and star formation aspects provided that two forms of dark matter exist: one, close to the visible gaseous form for explaining the properties of the spiral sequences as an evolutive sequence of dissipative gravitating disks, and one non-gaseous form for explaining the remaining "indestructible" dark mass in the evolved part of the sequence, the S0's and Sa's.

\section{The role of warps}

All these considerations have been made considering the plane dynamics of spiral galaxies, except for the bulge growth via vertical instabilities in the inner stellar disk.

But what about the dynamical effects transverse to the disks in the outer regions? Namely, a notorious puzzle in spiral galaxies is the ubiquitous warp phenomenon which has eluded a clear explanation up to now. For instance warps are unlikely to result from resonant normal modes, because the soft edges of galaxy disks damp discrete modes (Hunter \& Toomre, 1969). Normal modes generated by massive inclined dark halo (Dekel \& Shlosman, 1983; Sparke, 1984; Sparke \& Casertano, 1988) are ruled out by dynamical friction that damp the warp in a few dynamical times (Dubinski \& Kuijken, 1995). Only particular triaxial halos can produce a torque that leads to a warp with a straight line of node and negligible back reaction (Petrou, 1980). Interactions are efficient in warping disks (Hernquist, 1991; Huang \& Carlberg, 1997), however they cannot be invoked in isolated warped galaxies.

Warps are especially obvious in the HI outer disks, but to a lesser amplitude the stellar disks are also warped. Statistics of warps in HI (Bosma, 1991; Richter \& Sancisi, 1994; Garcia-Ruiz et al., 1998) and in the optical band (Reshetnikov \& Combes, 1998, 1999; Sanchez-Saavedra et al., 1990; SanchezSaavedra et al., 2003) reveal that more than half the spiral galaxies are warped and asymmetric. Warps are also linked to large scale disk horizontal asymmetries, both signatures showing that the outer spiral disks are not as well virialized as the inner optical disks.

To answer the question about the general cause of warps, we have first tried to well understand the dynamics of ideal isolated and purely self-gravitating

D R A F T Page 5 February 7, 2020, 5:59pm D R A F T 


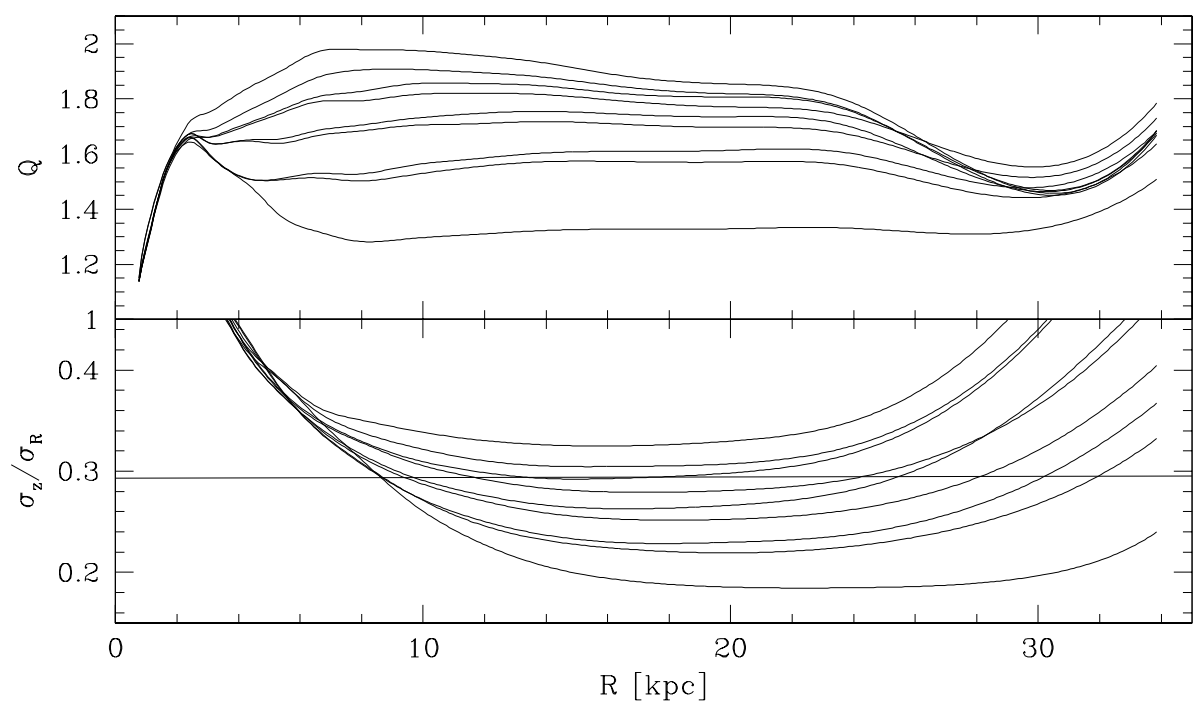

Figure 1. The radial stability parameter $Q$ (top) and the ratio $\sigma_{z} / \sigma_{R}$ (bottom) as a function of the galactic radius for different models. In both graphics, the curves correspond, from bottom to top, in the interval $R=10-20$, to the models of increasing thickness, respectively.

disks of collisionless particles. In a second step we will introduce energy dissipation, since disks form for the single reason that the energy dissipation rate is much faster than the angular momentum transport rate. Therefore energy dissipation must be taken as the second most important factor in understanding galaxies, after the pure gravitational dynamics of collisionless matter.

Consequently we have undertaken first to study in detail massive self-gravitating disks with various degrees of flattening by means of N-body simulations (Revaz \& Pfenniger, 2004). The simulated disks are made of a stellar bulge and an exponential disk components, and a collisionless heavy disk component proportional to the HI disk, including a density depression in the optical disk, and a flaring thickness almost proportional to radius. The Milky Way is the template galaxy for guiding the choice of the various mass ratios and scale lengths. The mass components and profiles are also such that an almost flat rotation curve are obtained for any thickness of the heavy disk component. By solving the Jeans equations separately for each mass component, we can start simulations with an almost equilibrium model, but with various degrees of velocity dispersion ratios $\sigma_{r} / \sigma_{z}(R)$, while keeping an initial Toomre parameter $Q$ well above 1 on almost the full radial range (see Fig. 1).

The main result is that conformally to predictions made long ago by Toomre (1966) and Araki (1985), too flat disks are unstable with respect to bending instabilities. The instability in thin sheets is just related to the velocity dispersion

D R A F T Page 6 February 7, 2020, 5:59pm D R A F T 


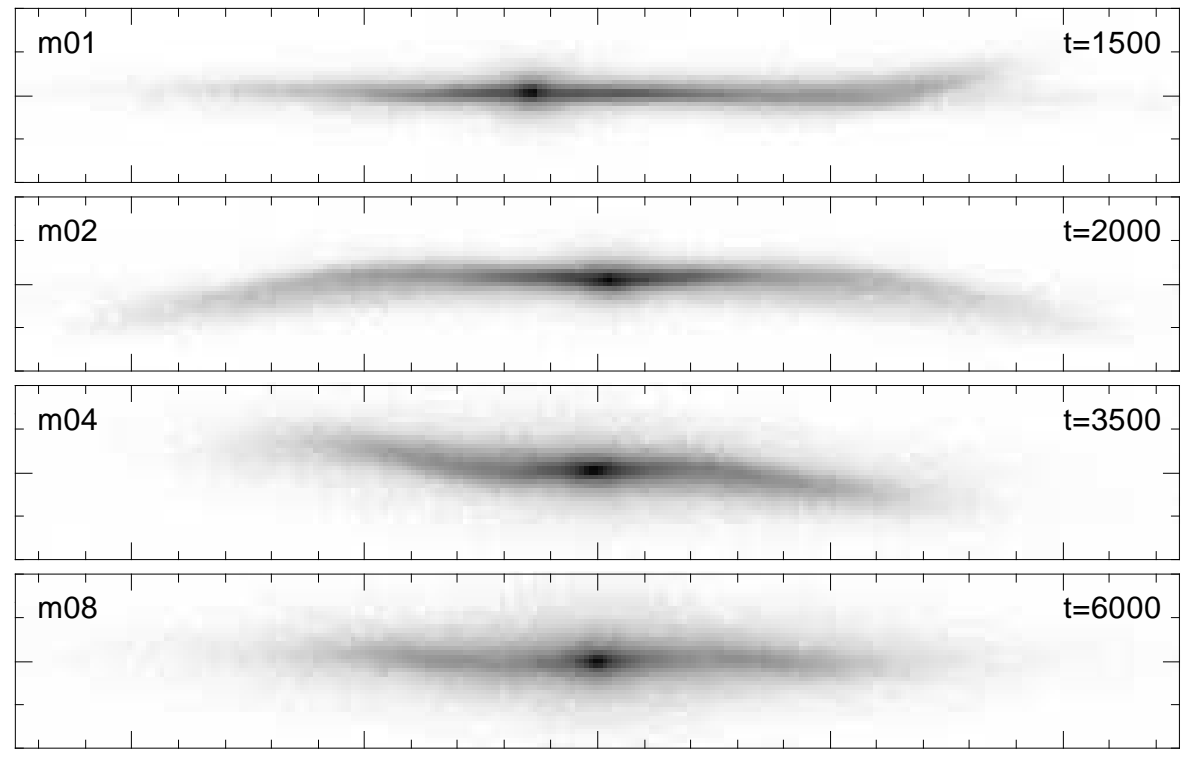

Figure 2. Edge-on projections of different heavy disk models having developed spontaneously a warp. The box dimensions are $100 \times 16 \mathrm{kpc}^{2}$

ratios between the vertical and radial velocity dispersions $\sigma_{z}$ and $\sigma_{R}$. When $\sigma_{z} / \sigma_{R}<0.293$ the sheet bends spontaneously with growth rates of order of Gyr. In thin disks this translates to first S-shaped warp growing modes for slightly unstable disks, and secondly for strongly unstable disks to U-shaped warp modes persisting for Gyrs (see Fig. 2).

If, like bars and spiral arms, warps results of internal disk instabilities, not only we obtain a unified picture of galaxies, but also several new clues about the dark matter nature and its distribution. For the same fundamental cause, the marginal stability of self-gravitating disks subject to an energy dissipation, disk produce spontaneously bars, spirals and warps that counteract dissipation by mechanical heating.

In order to be in such a warped state self-gravitating disks must first obviously have a dissipative component. Dusty gas can be identified as the primary cause of energy losses. Second, the mass distribution must be sufficiently selfgravitating and thin in order to reach the Araki stability threshold. This provides an interesting constraint for the gravitating mass.

The Araki criterion immediately tells us that a disk made of classical smooth gas would never be transversally unstable because the gas pressure would be isotropic. But a classical gaseous disk dissipating its heat decreases correspondingly its pressure, and inevitably after some time reaches the SafronovToomre radial instability threshold, at which point the subsequent non-linear

D R A F T Page 7 February 7, 2020, 5:59pm D R A F T 
evolution depends on the detailed microscopic physics of the gas. In the galaxy disk case we know that the interstellar medium is widely non-homogeneous, which means that the isotropic pressure assumption is not necessarily valid. We also know well the most visible result of the gas instability, star formation. Once star form they evolve as collisionless matter, and disks with such fluids are well known to evolve towards anisotropic dispersions with $\sigma_{z} / \sigma_{R}$ ratios of the order of 0.5 (Pfenniger \& Friedli, 1991; Huber \& Pfenniger, 2001).

Therefore the Araki criterion can only be met with a combination of collisionless matter property to be able to have rather large velocity dispersion anisotropy, and a dissipative component, which lowers over time faster the velocity dispersion in $z$ than in $R$. The dynamical heating produced radially by spirals and bars increases also the velocity anisotropy if the radial heating is inefficiently transferred transversally to the plane. The radial dynamics of disks indeed heats effectively through bars and spiral arms essentially the radial kinematics, maintaining it above $Q \sim 1$.

\section{The two types of dark matter}

So the ubiquitous existence of warps in disk galaxies is a strong hint that they are sufficiently massive and thin to be transversally unstable to warp modes, preferentially $\mathrm{S}$-shaped modes. U-shaped modes are also possible if a disk is driven sufficiently deep below Araki's threshold. If this is the case then we must have a substantial mass component almost as thin as HI-disks that behaves as collisionless matter for several rotational periods. In order to regenerate warps, dissipation is essential, without it a too anisotropic disk heats dynamically until a stable thicker state is reached.

If disks react to bending instabilities by kinematical heating transverse to the disk, then one must expect that warped disks are maintained close to the marginal state balancing gas dissipation with dynamical heating.

Then the question is whether such warps may constrain the traditional thick and hot dark halos made of collisionless matter. By adding a corresponding potentials to the initial N-body models, we have calculated up to which halo mass with given flattening a marginal state to bending would be kept (Revaz \& Pfenniger, 2004). It turns out that the effective disk thickness provides a strong constraint on the dark halo mass, but almost no constraint on its flattening. In all studied cases the exact halo flattening is very little constrained by the marginal stability state above a density flattening around $0.3-0.5$, which is anyway the range usually considered in cosmological simulations. In contrast, the relative mass of a hot dark halo within a radius comparable to the HI disk radius is directly related to the precise massive disk thickness: the thicker the marginally unstable disk is, the lesser mass can be contained in hot spheroidal

D R A F T Page 8 February 7, 2020, 5:59pm D R A F T 
thick halo assuming that the warp results from a bending instability. For models parameters fitted to the Milky Way, the halo is at most as heavy as the disk.

Since we can estimate the HI disk thickness, we can give a constraint of the dark halo mass if the disk dark matter has a thickness similar to the HI. The Milky Way has a known HI thickness and a known warp, therefore if this warp results from a disk marginal stability state then the dark halo mass within a radius similar to the HI disk radius $(\sim 30 \mathrm{kpc})$ is constrained to be below 0.4. This value is similar to the dark matter fraction found in evolved Sa's, S0's.

\section{Conclusions}

By realizing that bars, spirals and warps are different effects resulting from that same fundamental causes, a slightly energy dissipative gas component acting secularly on a mostly collisionless rotating self-gravitating system, we obtain new clues about dark matter.

First confirming several studies about the horizontal dynamics of galaxies, we arrive at the conclusion that spiral disks are to first order self-gravitating and collisionless, they must contain more matter than seen. This matter must be weakly collisional in order to develop anisotropic velocity dispersions in $R$ and $z$. It is clear that the radial velocity dispersion is rapidly regulated by the radial dynamical instabilities, so the anisotropy increases in $z$ through a dissipative component, identified with the dusty gas.

Dissipation must be sufficiently effective in order to maintain the disks close to instability, as witnessed by the spirals and warps, but also most of the mass cannot be strongly collisional, otherwise the velocity dispersion would be isotropic and no bending instability would occur. The physical solution that we are investigating is close to the clumpuscule model in Pfenniger \& Combes (1994), where much of the mass is condensed in the form of cold, dense planetmass molecular hydrogen clumps, stabilized in their core by a solid or liquid phase of molecular hydrogen (Pfenniger, 2004ab; Revaz \& Pfenniger, 2004b).

The warped N-body models do not rule out traditional massive thick halos with extended core. However the mass of the massive halo can hardly exceed the massive disk mass if this disk possesses a warp produced by a bending instability. The models do not constrain well the dark halo axis ratio, provided it is above $\sim 0.3$.

Thus we arrive at a bi-modal solution for dark matter in galaxies that seems to satisfy all the known constraints, from observational to galaxy dynamics and evolution constraints, and to cosmological constraints. Of course the nature of the dark matters remain to be better understood and discovered by observations. Encouragingly, tiny clumps of molecular hydrogen have been recently detected (Heithausen, 2004), but the nature of the hot, non cuspy dark halos remain to be found.

D R A F T Page 9 February 7, 2020, 5:59pm D R A F T 


\section{Acknowledgments}

This work has been supported by the Swiss National Science Foundation.

\section{References}

Araki, S. 1985, Ph.D. Thesis, Massachusetts Inst. Technology

Bosma, A. 1991, in : Warped disks and inclined rings around galaxies, Casertano S., Sackett P., Briggs F.H. (eds.), Cambridge University Press, 181

Bureau, M., Freeman, K.C. 1999, AJ, 118, 126

Combes F., Debbasch F., Friedli D., Pfenniger D. 1990, A\&A 233, 82

Combes F., Sanders R.H. 1981, A\&A 96, 164

Dekel A., Shlosman I. 1983, in : Internal Kinematics and Dynamics of Galaxies, E. Athanassoula (ed.), IAU 100, 177

Dubinski, J., Kuijken, K. 1995, ApJ, 442, 492

Elmegreen, B. 2004, astro-ph/0405555

Fleck R.C. 1981, APJ 246, L151

Garcia-Ruiz I., Kuijken K., Dubinski K. 1998, in : Galactic Halos: A UC Santa Cruz Workshop, D. Zaritsky (ed.), ASP Conference Series, 136, 385

Hernquist L. 1991, in : Warped disks and inclined rings around galaxies, Casertano S., Sackett P., Briggs F.H. (eds.), Cambridge University Press

Heithausen, A. 2004, ApJ, 606, L13

Huang, S., Carlberg, R.G. 1997, ApJ, 480, 503

Huber, D., Pfenniger, D. 2001, A\&A, 374, 465

Hunter, C., Toomre, A. 1969, ApJ, 155, 747

Klessen, R.S. 2004, astro-ph/0402673

Ostriker, J.P., Peebles, P.J.E. 1973, ApJ 186, 467

Petrou, M. 1980, MNRAS, 191, 767

Pfenniger, D. 2004a, in preparation

Pfenniger, D. 2004b, in The Dense Interstellar Medium in Galaxies, S. Pfalzner et al. (eds.) Springer, p. 409

Pfenniger, D., Combes, F. 1994, A\&A, 285, 91

Pfenniger, D., Combes, F., Martinet, L. 1994, A\&A, 285, 79

Pfenniger, D., Friedli, D. 1991, A\&A, 252, 7

Reshetnikov, V., Combes, F. 1998, A\&A, 337, 9

Reshetnikov, V., Combes, F. 1999, A\&AS, 138, 101

Revaz, Y., Pfenniger, D. 2004a, A\&A in press

Revaz, Y., Pfenniger, D. 2004b, A\&A in preparation

Richter, O.G., Sancisi, R. 1994, A\&A 290, L9-L12

Sanchez-Saavedra, M.L., Battaner, E., Florido, E. 1990, MNRAS, 246, 458

Sanchez-Saavedra, M.L., Battaner, E., Guijarro, A., et. al. 2003, A\&A, 399, 457

Sancisi, R. 2003, at IAU Symposium 220 "Dark Matter in Galaxies"

Sparke, L.S. 1984, MNRAS, 211, 911

Sparke, L., Casertano, S. 1988, MNRAS, 234, 873

Toomre, A. 1966, Geophys. Fluid Dyn., 46, 111 